\title{
Extensor Pollicis Longus Tendon Rupture after Non-Displaced Extra-Articular Distal Radius Fracture: A Case Report
}

\author{
Mohamed Tall*, Hervé Pilabre, Adama Ouedraogo, Alidou Porgo, Gnounsiniyapoué Bonkian \\ Department of Orthopedics and Traumatology, Bogodogo University Hospital of Ouagadougou, Ouagadougou, Burkina Faso \\ Email: *mohatall@yahoo.fr
}

How to cite this paper: Tall, M., Pilabre, H., Ouedraogo, A., Porgo, A. and Bonkian, G. (2020) Extensor Pollicis Longus Tendon Rupture after Non-Displaced Extra-Articular Distal Radius Fracture: A Case Report. Open Journal of Orthopedics, 10, 6-12. https://doi.org/10.4236/ojo.2020.101002

Received: November 1, 2019

Accepted: December 22, 2019

Published: December 25, 2019

Copyright ( 2020 by author(s) and Scientific Research Publishing Inc. This work is licensed under the Creative Commons Attribution International License (CC BY 4.0).

http://creativecommons.org/licenses/by/4.0/

\begin{abstract}
Background: Spontaneous tendon rupture of hand is not frequent. These ruptures can occur after a fracture. Aim: We report a case of spontaneous rupture of extensor pollicis longus tendon, and describe the treatment. Case presentation: We report the case of a 63-year-old woman who had extensor pollicis longus tendon rupture after non-displaced extra-articular distal radius fracture, treated by wrist circular cast immobilization. Extensor indicis proprius tendon transfer was performed, with an excellent functional result. Conclusion: Extensor pollicis longus tendon rupture can occur after non-displaced extra-articular distal radius fracture. Its treatment by tendon transfer helps to restore function of hand.
\end{abstract}

\section{Keywords}

Distal Radius Fracture, Tendon Rupture, Transfer

\section{Introduction}

Spontaneous tendons rupture of hand is not frequent. They are easy to diagnose and difficult to treat.

Spontaneous extensor pollicis longus tendon rupture is rare and often associated with systemic diseases [1]. Many cases of post-traumatic tendon rupture are related to a surgical treatment of the fracture with internal fixation [2].

These spontaneous ruptures and all the progressive ruptures secondary to tendon wear may require a graft or tendon transfer.

We report a case of extensor pollicis longus tendon rupture after orthopedic treatment of a non-displaced distal radius fracture in a patient with no previous medical history. 


\section{Observation}

A 63-year-old right-handed retired woman without any medical antecedent (any systemic diseases) was admitted in emergency for closed trauma of the right wrist. The diagnosis of non-displaced extra-articular distal radius fracture was made after radiography (Figure 1). An orthopedic treatment with immobilization by a wrist circular cast performed on the same day.

At the 14th day of immobilization this patient consult for impossibility of active extension of the thumb (Figure 2(a)), without limitation of the passive extension (Figure 2(b)).

Clinical examination (wrist pain next to the radial styloid, impossibility of active thumb extension, and no limitation of active extension), and ultrasound revealed a rupture of extensor pollicis longus tendon (Figure 2(c)).

Surgical treatment was decided. Under general anesthesia with a tourniquet on the arm and upper limb on a tablet, the approach centered on the path of the extensor pollicis longus tendon allowed to highlight a wear rupture on the bone roughness. It was a progressive rupture by friction of the tendon on the fracture site (Figure 3(a)). The condition of the extremities of the tendon does not allow a termino-terminal suture, tendon transfer was decided (Figure 3(b)). A second $2 \mathrm{~cm}$ incision next to the 2nd metacarpal allowed the section of the tendon of extensor indicis proprius and its transfer by termino-terminal suture to the distal end of the extensor pollicis longus tendon. The suture of the transfer was termino-terminal (Figure 4(a) and Figure 4(b)) according to the technique of Pulvertaft [3]. A wrist cast taking the thumb was set up postoperatively for a period of 45 days (Figure 5). Physiotherapy and rehabilitation were prescribed for six weeks after removal of cast.

At the 12-month follow-up, the functional evaluation found a good thumb function with normal active extension and a good hand clamping force measured at $20 \mathrm{~kg}$ at the Jamar dynamometer (Figure 6).

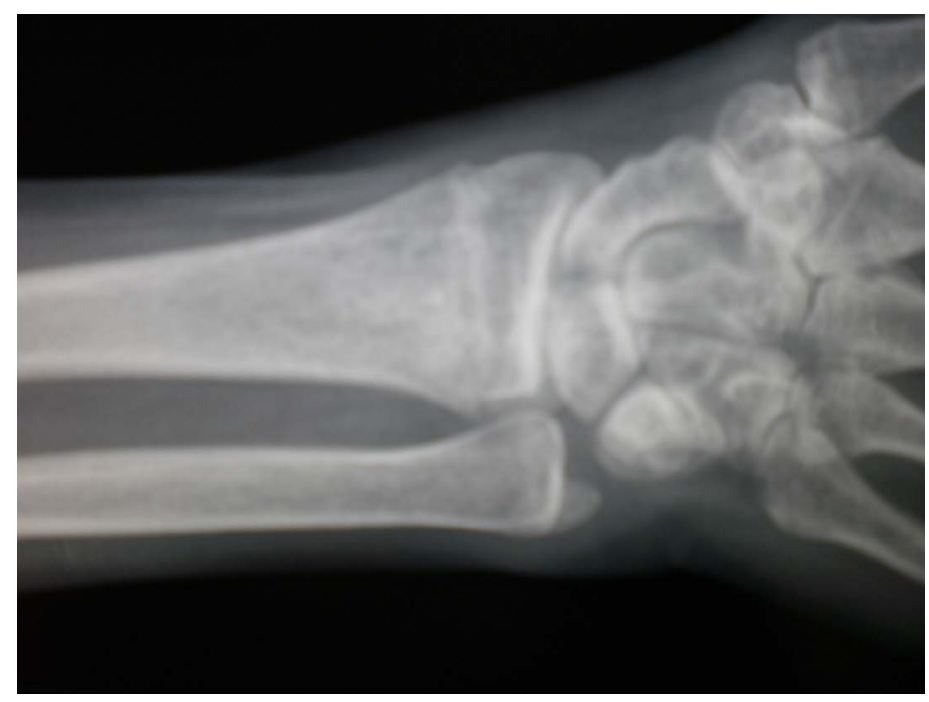

Figure 1. Radiography of non-displaced extra-articular distal radius fracture. 


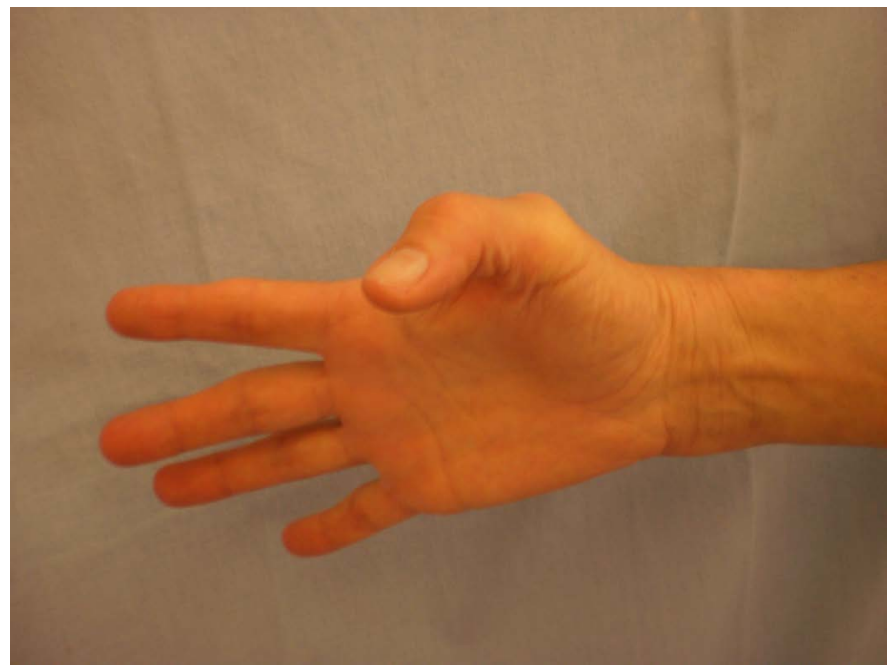

(a)

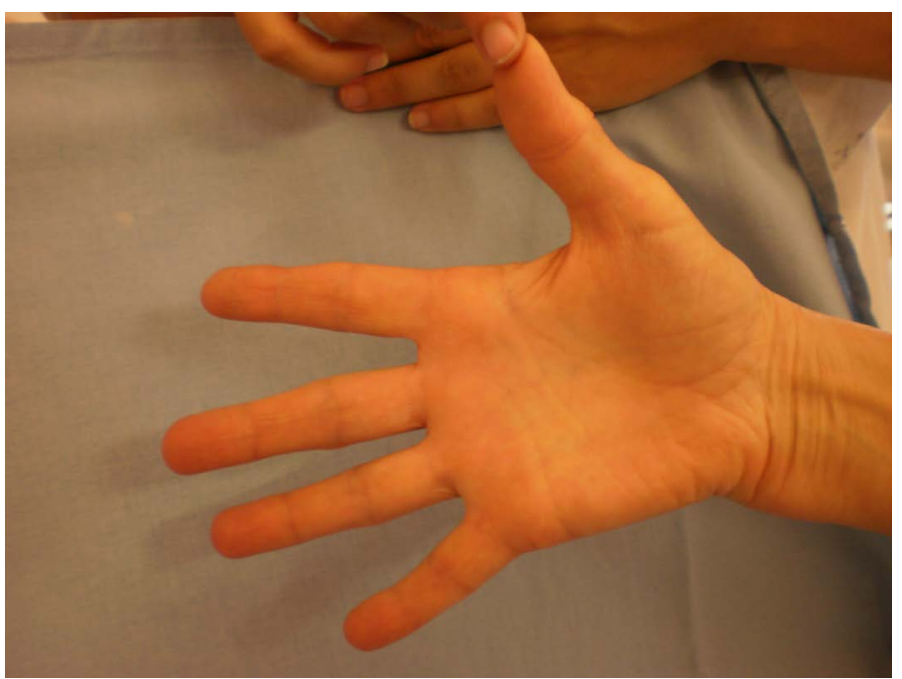

(b)

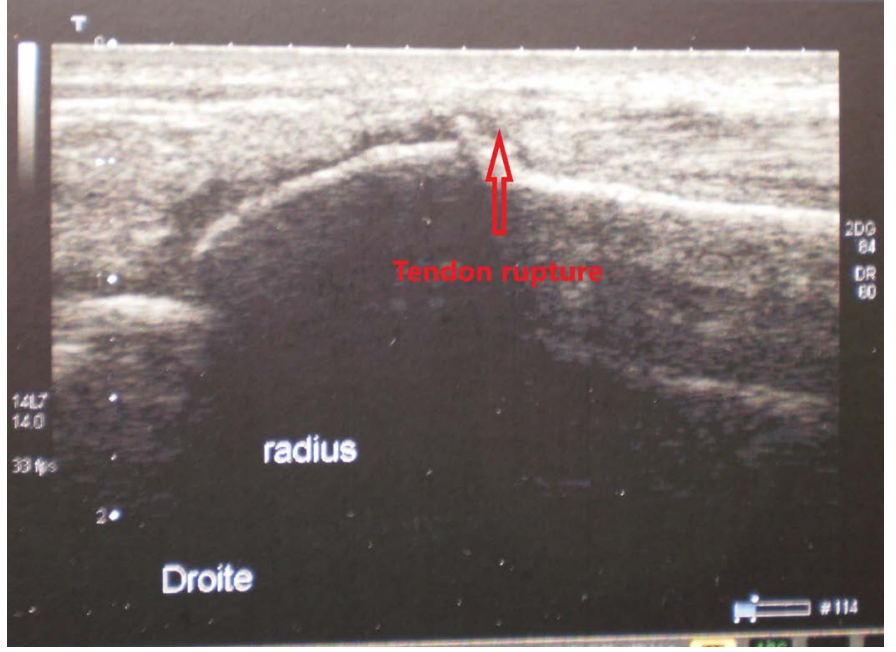

(c)

Figure 2. (a) Impossibility of active extension of the thumb; (b) No limitation of the passive extension; (c) Rupture of extensor pollicis longus tendon at ultrasound. 


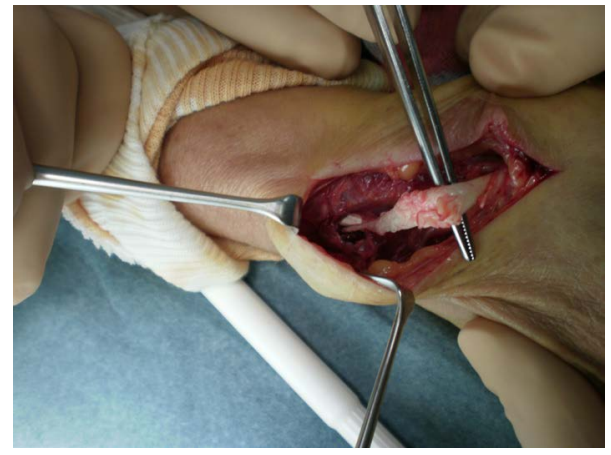

(a)

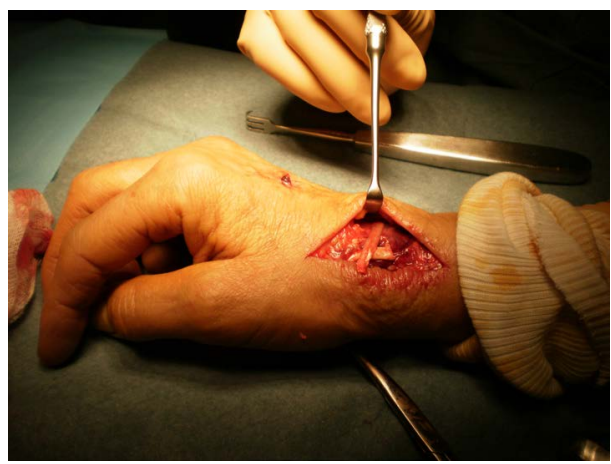

(b)

Figure 3. (a) Tendon rupture secondary to a friction on a relief at the distal radius fracture; (b) Transfer of the extensor indicis proprius tendon.

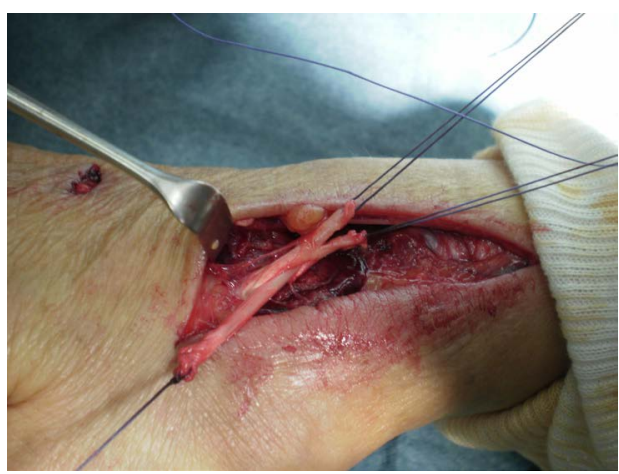

(a)

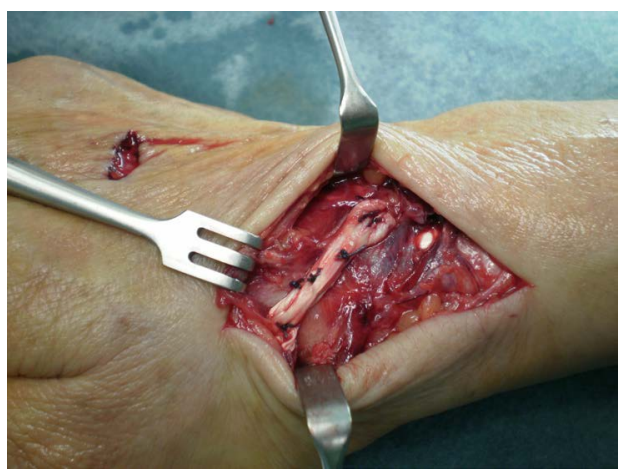

(b)

Figure 4. (a) and (b) Pulvertaft termino-terminal suture of the tendon transfer. 


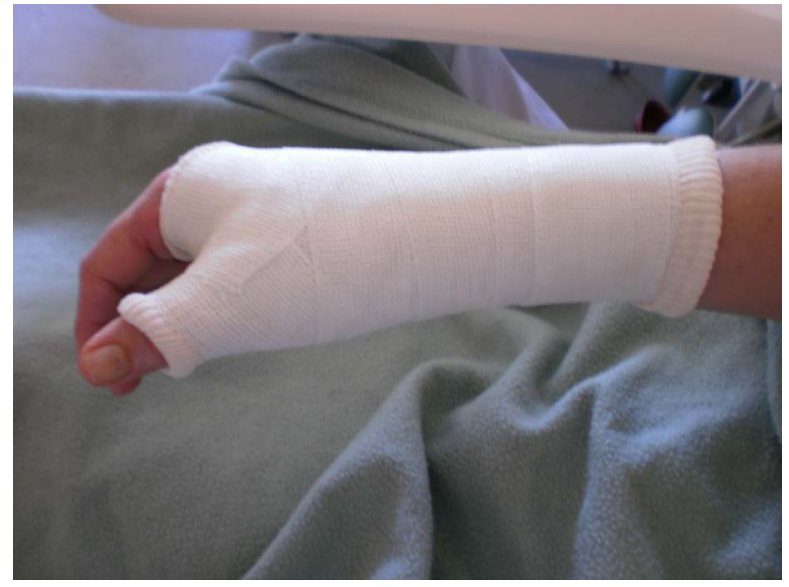

Figure 5. Immobilization of wrist and thumb by circular cast.

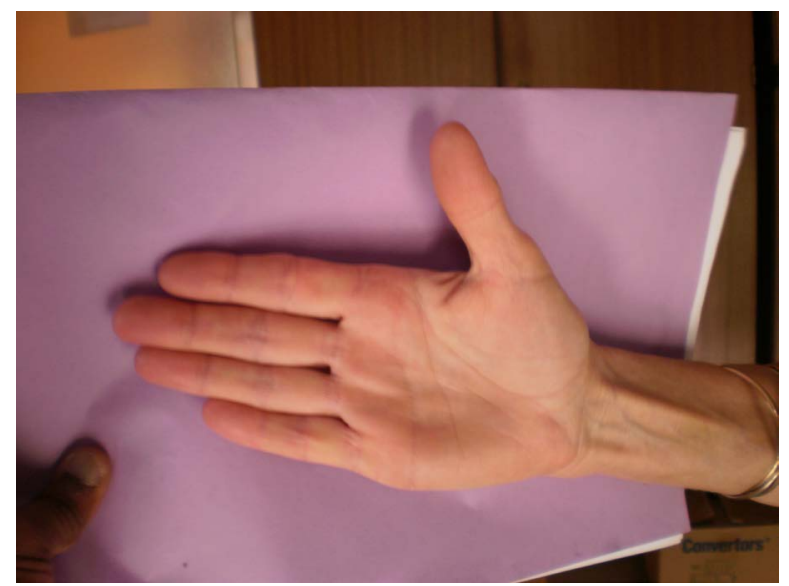

Figure 6. Good thumb function with normal active extension.

\section{Discussion}

The extensor pollicis longus tendon rupture after a distal radius fracture has been reported in the literature and several traumatic [2] and non-traumatic etiologies are described [1]. The most common tendon rupture are those related to the presence of osteosynthesis material [2].

The tendon rupture in this case was secondary to a friction on a roughness of the fracture site. Friction on another type of roughness such as a sesamoid bone can also lead to tendon rupture [4]. This same mechanism of friction can be linked to an osteophyte on osteoarthritis of carp. The rupture was secondary to attrition caused by a bony protrusion of advanced trapeziometacarpal joint osteoarthritis [5].

The progressive tendon lesion did not allow repair by tendinous suture. Tendon transfer of the extensor indicis proprius was performed. The Pulvertaft technique [3] allowed us to suture two tendons of different size and shape. This allows to obtain a resistant suture if it is well practiced [6] [7]. Although less resistant than cow-hitch technique [8] we opted for the Pulvertaft technique because of easier realization in our case. 
Transfer of the extensor indicis proprius tendon that we realized allowed to obtain an excellent result. Similar results were reported by Magnussen [9] and Winckler [10] who performed the same technique. For these authors it is a simple and reliable procedure with few complications [9] [10].

\section{Conclusion}

Non or Slightly displaced fractures of the distal radius can be a risk for tendons. These can conflict with a bone fragment. In case of non-suturable tendon rupture, transfer is a reliable surgical technique.

\section{Ethics Approval and Consent to Participate}

We obtained the informed consent from the patient to report this case.

\section{Human and Animal Rights}

No animals/humans were used for studies that are base of this research.

\section{Acknowledgements}

All authors contributed to proofreading and editing the manuscript before submission.

\section{Conflicts of Interest}

The authors declare no conflict of interest, financial or otherwise.

\section{References}

[1] Spar, I. (1977) Flexor Tendon Ruptures in the Rheumatoid Hand: Bilateral Flexor Pollicis Longus Rupture. Clinical Orthopaedics and Related Research, No. 127, 186-188. https://doi.org/10.1097/00003086-197709000-00026

[2] Cho, N.Y., Seo, C.Y., Kim, M.S., Kim, H.S. and Le, K.B. (2012) Extensor Pollicis Longus Rupture after Distal Radius Fracture. Journal of the Korean Fracture Society, 25, 52-57. https://doi.org/10.12671/jkfs.2012.25.1.52

[3] Pulvertaft, R.G. (1956) Tendon Grafts for Flexor Tendon Injuries in the Fingers and Thumb; a Study of Technique and Results. The Journal of Bone and Joint Surgery, 38-B, 175-194. https://doi.org/10.1302/0301-620X.38B1.175

[4] Webb, J.B. and Elliot, D. (1995) Spontaneous Rupture of the Flexor Pollicis Longus Tendon on a Sesamoid Bone. Journal of Hand Surgery, 22-B, 381-382.

[5] Morizaki, Y., Ohe, T., Kamekura, S. and Takamure, H. (2008) Rupture of the Extensor Pollicis Longus Tendon in Trapezio-Metacarpal Joint Osteoarthritis: Case Report. Journal of Hand Surgery, 33, 1179-1181. https://doi.org/10.1016/j.jhsa.2008.03.017

[6] Kannan, S., Ghosh, A.I., Dias, J.J. and Singh, H.P. (2019) Comparative Biomechanical Characteristics of Modified Side-to-Side Repair and Modified Pulvertaft Weaving Repair-In vitro Study. The Journal of Hand Surgery (Asian-Pacific Volume), 24, 76-82. https://doi.org/10.1142/S2424835519500140

[7] Duprat, A., Gayet, L.E., Breque, C. and Freslon, M. (2018) Biomechanical Study Comparing Pulvertaft Suture to Step-Cut Suture. Hand Surgery and Rehabilitation, 
37, 24-29. https://doi.org/10.1016/j.hansur.2017.11.004

[8] Vincken, N.L., Lauwers, T.M. and van der Hulst, R.R. (2017) Biomechanical and Dimensional Measurements of the Pulvertaft Weave versus the Cow-Hitch Technique. $H A N D, 12,78-84$. https://doi.org/10.1177/1558944716646758

[9] Magnussen, P.A., Harvey, F.J. and Tonkin, M.A. (1990) Extensor Indicis Proprius Transfert for Rupture of the Extensor Pollicis Longus Tendon. The Journal of Bone and Joint Surgery, 72-B, 881-883. https://doi.org/10.1302/0301-620X.72B5.2211775

[10] Winckler, S., Westphal, T. and Brug, E. (1995) Transposition of the Extensor Indicis Tendon in Reconstruction of Thumb Extension after Rupture of the Extensor Pollicis Longus Tendon. Vol. 66, Der Chirurg, 507-512. 